\title{
Modular invariance, universality and crossover in the quantum Hall effect
}

\author{
Brian P. Dolan \\ Department of Mathematical Physics, National University of Ireland, Maynooth, Ireland \\ Dublin Institute for Advanced Studies, 10 Burlington Road, Dublin, Ireland
}

Received 29 September 1998; revised 22 April 1999; accepted 27 May 1999

\begin{abstract}
An analytic form for the conductivity tensor in crossover between two quantum Hall plateaux is derived, which appears to be in good agreement with existing experimental data. The derivation relies on an assumed symmetry between quantum Hall states, a generalisation of the law of corresponding states from rational filling factors to complex conductivity, which has a mathematical expression in terms of an action of the modular group on the upper-half complex conductivity plane. This symmetry implies universality in quantum Hall crossovers. The assumption that the $\beta$-function for the complex conductivity is a complex analytic function, together with some experimental constraints, results in an analytic expression for the crossover, as a function of the external magnetic field. (C) 1999 Elsevier Science B.V. All rights reserved.
\end{abstract}

PACS: $11.10 . \mathrm{Hi} ; 73.40 . \mathrm{Hm}$

Keywords: Quantum Hall effect; Modular group; Renormalisation group flow; Critical point; Crossover

\section{Introduction}

The significance of symmetry groups in the understanding of physics, particularly quantum physics, has steadily increased ever since their importance was first realised. For example the representation theory of the rotation group in three dimensions is immensely powerful in understanding the structure of the periodic table of the elements, even before any underlying dynamics is studied, and the importance of symmetry principles in elementary particle physics cannot be over emphasised. It has been suspected for some time now [1-4] that a certain infinite discrete group, the modular group $\Gamma(1)$,

\footnotetext{
' E-mail: bdolan@thphys.may.ie
} 
acting on the upper-half complex conductivity plane, $\sigma=\sigma_{x y}+i \sigma_{x x}$, plays an important rôle in understanding the phase structure of quantum Hall $(\mathrm{QH})$ states. In particular the fact that only odd denominator filling factors, $\nu=p / q$ where $q$ is an odd integer, are observed as stable quantum Hall states (for which $\sigma_{x x}$ vanishes and $\sigma_{x y}=p / q$ ) can be interpreted as being due a symmetry between states under the action of a particular sub-group of the full modular group, often denoted by $\Gamma_{0}(2)$ in the literature [2,5]. It is the purpose of this paper to explore some of the consequences of this observation for the crossover from one $\mathrm{QH}$ state to another.

If one assumes that the renormalisation group (RG) flow in the $\sigma$ plane, induced by changing the strength of the external magnetic field, commutes with the action of $\Gamma_{0}(2)$, one is led to conclude that fixed points of $\Gamma_{0}(2)$ must be critical points of the RG flow, $[2,5]$. This leads to many predictions concerning the phase structure, including the selection rule for $\mathrm{QH}$ transitions $p_{1} q_{2}-p_{2} q_{1}= \pm 1$, between filling factors $\nu_{1}=p_{1} / q_{1}$ and $\nu_{2}=p_{1} / q_{2}[6]$. In Refs. [2,5,6] only the critical points and phase structure were discussed, nothing was said about the form of the crossover between QH sates beyond the observation that $\Gamma_{0}(2)$ leads naturally to the semi-circle law of [7] and follows from the law of corresponding states in [8]. Some suggestions about the functional form of the crossover were made in [9].

In this work some assumptions will be made about the analytic structure of the $\beta$ function for crossover between the $\mathrm{QH}$ state with $\nu=1$ and the insulating phase with $\nu=0$. Using this as a template one can then construct the $\beta$-function for crossover for any allowed $\mathrm{QH}$ transition, by acting with the appropriate element of $\Gamma_{0}(2)$ which maps $\nu=0$ and $\nu=1$ onto $\nu=p_{1} / q_{1}$ and $\nu=p_{2} / q_{2}$ respectively, with $p_{2} q_{1}-p_{1} q_{2}=1$. The validity of the assumptions can then be tested by comparing the resulting predictions with existing experiments, and the results are promising.

The most important assumption, as described in Section 3 , is that that the $\beta$-function is a complex analytic function of $\sigma$. Together with symmetry under the action of $\Gamma_{0}(2)$ this restricts the structure of the $\beta$-function to a very special form and some further, rather mild, assumptions restrict it to an essentially unique function. Unfortunately there is as yet no microscopic justification for the assumption that the $\beta$-function is analytic, rather the motivation comes form the apparently unrelated area of Yang-Mills gauge theory in four dimensions (for which the $\beta$-function was investigated in $[10,11]$, and much of the analysis presented here is based on the ideas in these references)--the rational here is that the modular group seems to be a common feature of both the quantum Hall effect and supersymmetric Yang-Mills theory so it may prove instructive to push the analogy as far as possible, even though a microscopic understanding is lacking at present. Ultimately experiment is the arbitrator and if pursuing this analogy gives predictions which disagree with the experimental results then it is clearly not a useful analogy. The functional form of the crossover resulting from the assumption of complex analyticity is shown in Fig. 3 for the transition from $\nu=1$ to the insulating phase, and in Fig. 4 for the crossover between $\nu=2$ and $\nu=1$, at various temperatures (the conductivity is expressed in multiples of $e^{2} / h$ ). The similarities between the theoretical predictions shown in Figs. 3 and 4 here and the experimental results, shown in Figs. 1 


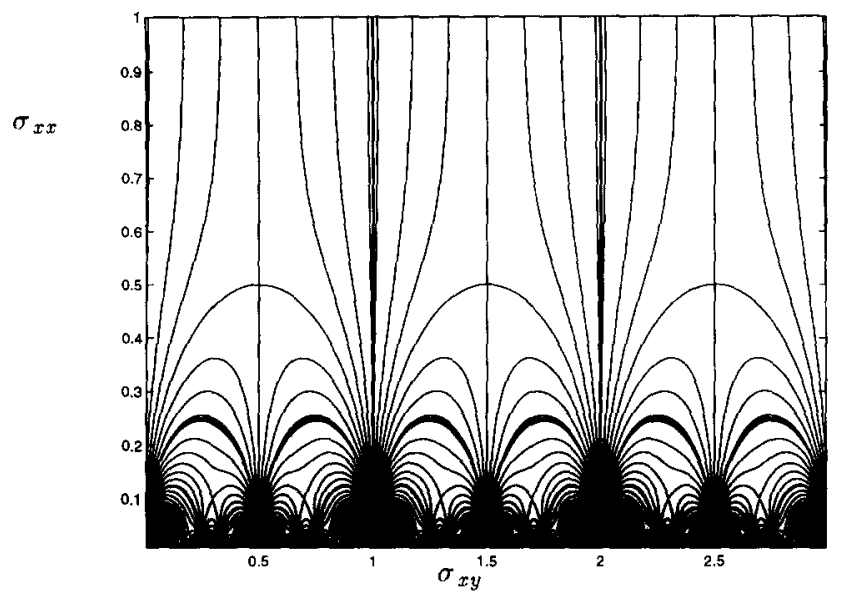

Fig. 1. RG flow in the complex conductivity plane for the quantum Hall effect. The thicker lines are cuts where the phase of $f(\sigma)$ jumps form $-\pi$ to $\pi$.

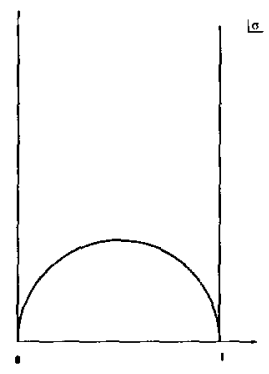

Fig. 2. The fundamental domain for $\Gamma_{0}(2)$ is the vertical strip of unit width above the semi-circle of unit diameter centred at $\sigma=1 / 2$.

and $2 b$ of [12] are quite striking, so it seems that the ansatz of a holomorphic $\beta$ function may indeed be useful. The lack of a microscopic justification is nevertheless an unsatisfactory feature of the analysis and, if the results presented here should prove to be useful in describing the quantum Hall effect, it is crucial that a microscopic justification be found-work on this is ongoing at the moment.

For ease of reference we give here the analytic form of the function which is postulated to describe crossover between QH states $\nu=p_{1} / q_{1}$ and $\nu=p_{2} / q_{2}$, which is the main result of this paper. Let $\Delta \nu=\nu-\nu_{c}$, with $\nu=\bar{n} e / B$ the Landau level filling factor $(\bar{n}$ is the electron density and $B$ the external magnetic field) and $\nu_{c}=\bar{n} e / B_{c}$ the filling factor at the critical value of the magnetic field, $B_{c}$. Define $\alpha:=\left(p_{2}-p_{1}\right)-\left(q_{2}-q_{1}\right) \nu_{c}$ and the linear function $\zeta(\Delta \nu):=\alpha\left\{\left(q_{1}-q_{2}\right) \Delta \nu+\alpha\right\}$. Then

$$
\sigma(\Delta \nu)=\frac{p_{2} q_{2}\left\{K^{\prime}(w)\right\}^{2}+p_{1} q_{1}\{K(w)\}^{2}+i K^{\prime}(w) K(w)}{\left[q_{1}^{2}\{K(w)\}^{2}+q_{2}^{2}\left\{K^{\prime}(w)\right\}^{2}\right]}
$$

where $K^{\prime}(w)$ and $K(w)$ are complete elliptic integrals of the second kind-with modulus $w$ a function of $\Delta \nu$ given by 


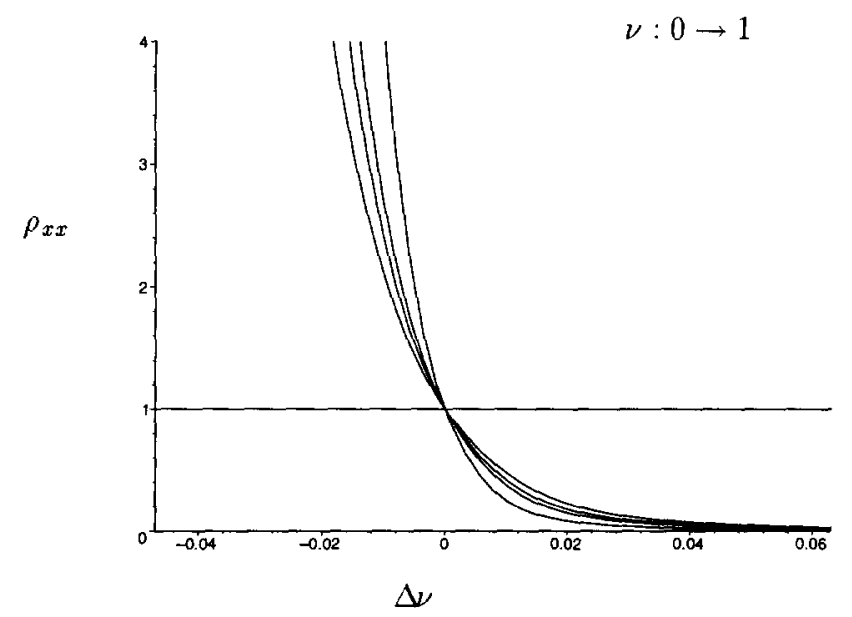

Fig. 3. Crossover of longitudinal resistivity for the insulator-QH transition $\nu: 0 \rightarrow 1$ at the four temperatures $T=42,84,106$ and $145 \mathrm{mK}$ with $A=60$ and $\mu=0.50$. To be compared with the experimental data in Fig. $2 \mathrm{~b}$ of $[12]$.

$$
w^{2}=\frac{1}{2}\left\{1-\operatorname{sign}(\Delta \nu) \sqrt{1-\mathrm{e}^{-\left(A \Delta \nu / \zeta(\Delta \nu) T^{\mu}\right)^{2}}}\right\} .
$$

As usual the complementary modulus $w^{\prime}$ is defined by $\left(w^{\prime}\right)^{2}=1-w^{2}$ and $K^{\prime}(w)=$ $K\left(w^{\prime}\right) . T$ in Eq. (2) denotes the temperature and $\mu$ the critical exponent described in [13], which is experimentally determined to be $\mu=0.45 \pm 0.05$ [12] (the definition is given in Section 2). $A$ is a positive real constant, possibly depending on other experimental parameters such as the electron density and $B_{c}$. The arguments that lead to Eq. (2) are strictly only valid for small $\Delta \nu$, in principle there could be corrections of order $(\Delta \nu)^{4}$ to the exponent, however the range of $\Delta \nu$ required for crossover is small, $|\Delta \nu|<0.1$, and Eqs. (1) and (2) appear to give a good fit to the experimental data as they stand-the crossover that they describe is shown graphically in Figs. 3 and 4. Fig. 3 is obtained by plotting the real and imaginary parts of (1) for the case $p_{1}=0$, $p_{2}=q_{1}=q_{2}=1$ and Fig. 4 for $p_{1}=q_{1}=q_{2}=1, p_{2}=2$, and these figures should be compared with the experimental data in Figs. 1 and $2 b$ of Ref. [12]. For the special case of the $\mathrm{QH}$-insulator transition with $p_{1}=0$, and $q_{1}=q_{2}=p_{2}=1 \mathrm{Eq}$. (1) gives a resistivity, $\rho=-1 / \sigma$, whose longitudinal component is

$$
\rho_{x x}(\Delta \nu)=\frac{K(w)}{K^{\prime}(w)}
$$

from which follows the relation

$$
\rho_{x x}(\Delta \nu)=\frac{1}{\rho_{x x}(-\Delta \nu)},
$$

for this transition, since $w(-\Delta \nu)=w^{\prime}(\Delta \nu)$. This relation is well supported experimentally [14]. It seems remarkable that making the simplest possible assumption at every stage leads to predictions which are so close to experiment and the bulk of this paper is 

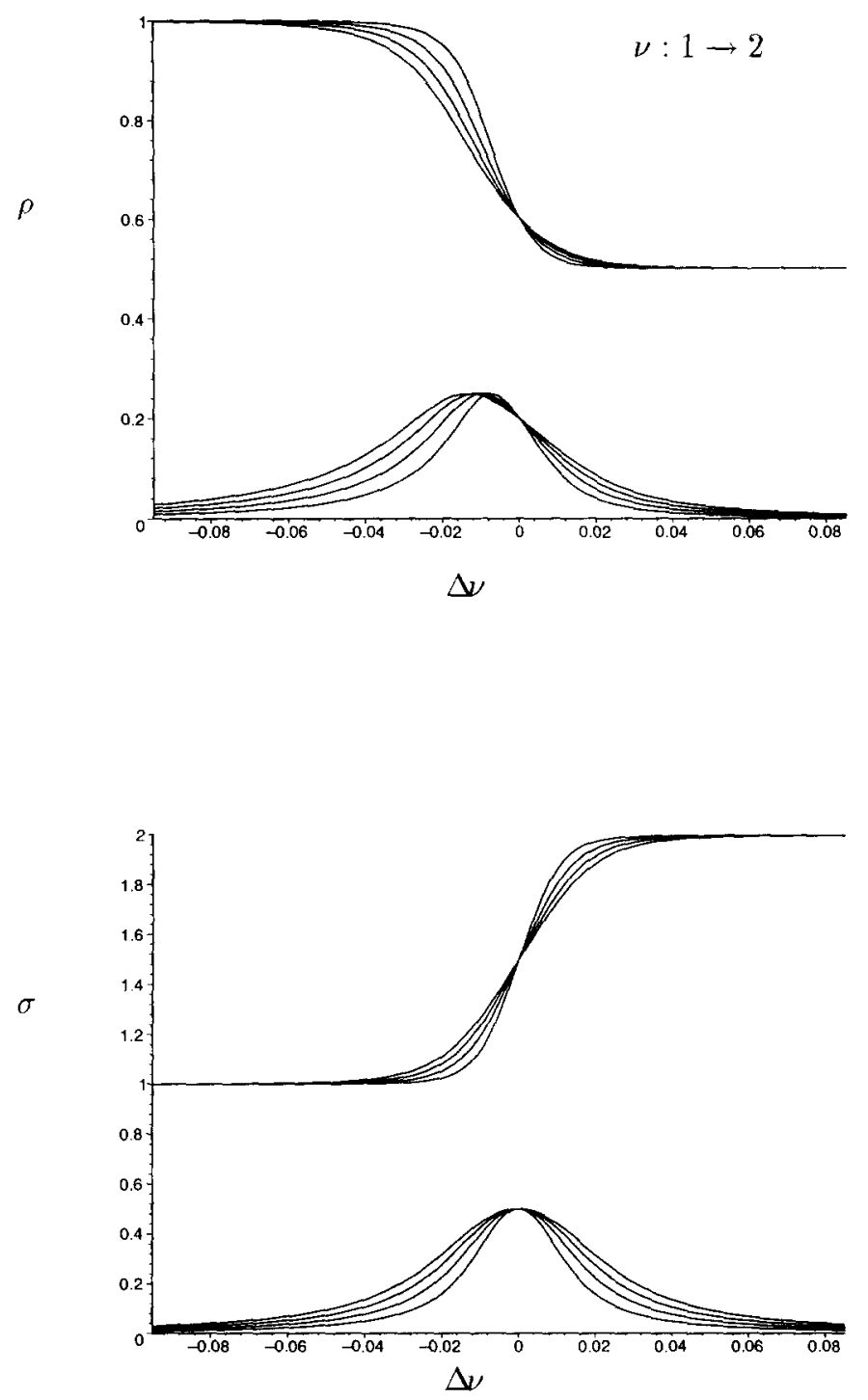

Fig. 4. Crossover of conductivity and resistivity for $\nu: 1 \rightarrow 2$ at the four temperatures $T=42,70,101$ and $137 \mathrm{mK}$ with $A=40$ and $\mu=0.50$. To be compared with the experimental data in Fig. 1 of $[12]$.

devoted to the derivation of Eq. (1). Explicitly, the assumptions which are used in the analysis are

(i) The law of corresponding states of [8] should be extended into the upper-half complex conductivity plane, $\sigma=\sigma_{x y}+i \sigma_{x x}$ (in units of $e^{2} / h$ ). This is related to rotational invariance in the bulk and is encoded mathematically into a group action on $\sigma$-specifically the group is $\Gamma_{0}(2)$.

(ii) The action of $\Gamma_{0}(2)$ commutes with $\mathrm{RG}$ flow. This implies universality of $\mathrm{QH}$ transitions. 
(iii) There are no extra critical points, other than those already indicated by the experimental data and weak coupling expansions, i.e. $\sigma=0,1, \frac{1+i}{2}, i \infty$ and their images under the action of $\Gamma_{0}(2)$.

(iv) In the limit of large $\sigma_{x x}$ (the weak coupling limit of field theoretical models) $\sigma_{x y}$ does not run and the $\beta$-function for $\sigma_{x x}$ is independent of $\sigma_{x y}$, finite and negative.

(v) The $\beta$-functions for the RG flow that describes crossover between two $\mathrm{QH}$ plateaux, or between a $\mathrm{QH}$ state and the insulating phase, are complex analytic functions when described in terms of a real variable $s$ that is a monotonic real analytic function of the external magnetic field.

These assumptions alone actually lead to slightly more general form than Eq. (1), but demanding that Hall plateaux are approached as fast as possible as the magnetic field is varied gives Eq. (1).

In the next section the concept of universality and scaling in the $\mathrm{QH}$ effect is reviewed and Section 3 contains a discussion of the rôle of $\Gamma_{0}(2)$ in $\mathrm{QH}$ phenomena. Section 4 then introduces the assumption of analyticity into the discussion and it is shown how this places strong restrictions on the form of the $\beta$-function. Section 5 continues with a discussion of the RG behaviour near the critical point in the crossover between Hall plateaux and it is shown that the $\beta$-function is then essentially unique. In Section 6 the analytic form of the crossover is discussed and Eq. (1) is derived. Finally Section 7 gives a summary and outlook. Appendix A contains a review of some properties of the modular group, Jacobi $\vartheta$-functions and complete elliptic integrals which are relevant to the discussion in the text but may be unfamiliar to a general audience.

\section{Scaling and universality in the quantum Hall effect}

The discussion of crossover relies heavily on the scaling analysis of [13], building on ideas originally due to Khmel'nitskii [15]. This section is a review of the essential points necessary for the later analysis. In [13] it was suggested that there was a critical point in the crossover between two QH states $\sigma=\nu_{1}$ and $\sigma=\nu_{2}$, near which the only relevant variables are the external magnetic field, $B$, and the temperature, $T$. The crossover is driven by varying $B$ at fixed $T$, and not by varying $T$ as in the more familiar temperature driven transitions (for a review of quantum phase transitions see [16]). The critical external field will be denoted by $B_{c}$. Thus the correlation length diverges with critical exponent $\nu_{\xi}, \xi \propto\left|B-B_{c}\right|^{-\nu_{\xi}}$ (the subscript $\xi$ on the exponent is to avoid confusion with the Landau level filling factor, which is also traditionally denoted by $\nu$ and appears frequently throughout the text). Since $\sigma$ is dimensionless, in units of $e^{2} / h$, it must be a function of a dimensionless ratio, $\tilde{b}=\Delta B / T^{\mu}$, where $\Delta B=B-B_{c}$ and $\mu$ is a critical exponent which appears to be universal in that it is the same for all transitions. The correlation length, $\xi$, is related to $\tilde{b}$ through a characteristic length, $L$. For low magnetic field strengths $L$ can be taken to be the electron scattering length [15], which diverges as $T \rightarrow 0$ for an infinite size system. The scaling form for the way in which electron scattering length depends on the temperature is [13] 


$$
L(T) \propto T^{-p / 2}
$$

where $p$ is the inelastic scattering length exponent. For high field strengths a more appropriate characteristic length scale is the magnetic length, $\sqrt{\hbar / e B}$. In any case $L$ would be expected to be a function of both $T$ and $B$ in general.

Now the ratio $l=L / \xi$ is dimensionless and requiring that the correlation length is related to the magnetic field by the usual scaling relation

$$
\xi \propto|\Delta B|^{-\nu_{\xi}}
$$

one has $|\tilde{b}| \propto l^{1 / \nu_{\xi}}$. Experimentally $\mu=0.45 \pm 0.05[12]$ and $\nu_{\xi}=2.3 \pm 0.1$.

The analytic from of the crossover is then described by a function $\sigma(\tilde{b})$ and the $\beta$ functions for the longitudinal and transverse conductivities $\sigma_{x x}$ and $\sigma_{x y}$ can be defined as

$$
\beta_{x x}\left(\sigma_{x x}, \sigma_{x y}\right)=\frac{d \sigma_{x x}}{d \tilde{b}}, \quad \beta_{x y}\left(\sigma_{x x}, \sigma_{x y}\right)=\frac{d \sigma_{x y}}{d \tilde{b}} .
$$

These can be combined into a single complex function $\beta=\beta_{x y}+i \beta_{x x}$ so that

$$
\beta(\sigma, \bar{\sigma})=\frac{d \sigma}{d \tilde{b}}
$$

Very little is known about the functional form of (7). A flow diagram was suggested in [15] and used in [13]. Asymptotic forms for $\sigma_{x x}$ and $\sigma_{x y}$ when $\sigma_{x x}$ is large have been calculated in [17], based on an effective action which contains a topological term. The interaction strength is $1 / \sigma_{x x}$, so that large $\sigma_{x x}$ is the weak coupling regime, and the topological term has coupling $\sigma_{x y}$, the quantization of which explains the quantization of the Hall states. In a perturbative calculation, $\sigma_{x y}$ is constant and $\beta_{x x}$ depends only on $\sigma_{x x}$, but it is argued in [17] that non-perturbative instanton effects produce the more general form (7). The same is true for supersymmetric Yang-Mills gauge theories, as revealed by the work of Seiberg and Witten [18], on which Refs. [10,11] are based. Ref. [18] itself relied heavily on a conjecture that the modular group should be a symmetry for supersymmetric Yang-Mills theory [19]. A non-perturbative form for $\beta(\sigma, \bar{\sigma})$, based on $\Gamma_{0}(2)$ symmetry, was suggested in [9].

In the article by Pruisken in [17] the $\beta$-functions are calculated as derivatives with respect to $l$ rather than $\tilde{b}$-this is quite legitimate and just reflects the fact that there are different ways to define $\beta$-functions. Another possibility is to use the Landau level filling factor $\nu=\bar{n} e / B$, where $\bar{n}$ is the electron density. Clearly

$$
\Delta \nu:=\nu-\nu_{c}=-\frac{\bar{n} e \Delta B}{B_{c}\left(B_{c}+\Delta B\right)} \approx-\frac{\bar{n} e \Delta B}{\left(B_{c}\right)^{2}},
$$

where $\nu_{c}=\bar{n} e / B_{c}$ is the critical filling factor, and $\beta$-functions could be defined using $v:=\bar{n} e \Delta \nu / T^{\mu}$ as

$$
\beta(\sigma, \vec{\sigma})=\frac{d \sigma}{d v}
$$

In the following we shall try to be as general as possible and define 


$$
\beta(\sigma, \bar{\sigma})=\frac{d \sigma}{d s}
$$

where $s$ is a monotonic function of $\tilde{b}$ or $v$, to be determined.

\section{The modular group and the quantum Hall effect}

This section reviews the essential ingredients of the action of the group $\Gamma_{0}(2)$ on the complex conductivity plane-more details can be found in Appendix A. The full modular group, $\Gamma(1)$, was studied for possible significance in the QH effect in [1], inspired by observations originally due to Cardy and Rabinovici [20] concerning the extension of Kramers-Wannier duality to a model with two parameters, one of which was topological in nature. Sub-groups of the modular group, resulting in a more restrictive symmetry, were analysed in Refs. [2-5], and in Refs. [2,5] attention was focused on the particular group $\Gamma_{0}(2)$, which was further developed in Ref. [6].

The basic assumption is that the infinite discrete group $\Gamma_{0}(2)$ has a natural action on the upper-half complex conductivity plane, parameterised by $\sigma=\sigma_{x y}+i \sigma_{x x}$ in units of $e^{2} / h$, which is a symmetry of the partition function (a generalisation of KramersWannier duality) and commutes with the RG flow on the $\sigma$-plane. The group $\Gamma_{0}(2)$ can be represented by $2 \times 2$ matrices of the from

$$
\left(\begin{array}{cc}
a & b \\
2 c & d
\end{array}\right)
$$

where $a, b, c$ and $d$ are integers with $a d-2 b c=1$ (which requires $a$ and $d$ to be odd). For

$$
\gamma=\left(\begin{array}{cc}
a & b \\
2 c & d
\end{array}\right) \in \Gamma_{0}(2)
$$

the action is $\gamma: \sigma \rightarrow(a \sigma+b) /(2 c \sigma+d)$. Thus $\gamma$ maps the $\mathrm{QH}$ state with filling factor $\nu=p / q$, which has $\operatorname{Re}(\sigma)=\nu$ and $\operatorname{Im}(\sigma)=0$, to $\nu=(a p+b q) /(2 c p+d q)$. Note that $2 c p+d q$ is odd if $q$ is, but this would not be true if the factor of 2 were omitted-this is one way of seeing why it is the sub-group $\Gamma_{0}(2)$ that is relevant to the $\mathrm{QH}$ effect rather than the full modular group, for which the 2 would not be present.

All $\Gamma_{0}$ (2) transformations can be generated by repeated applications of the two operators $U: \sigma \rightarrow \sigma+1$ and $V: \sigma \rightarrow \sigma /(2 \sigma+1)$, the former being a generalisation of the Landau level addition transformation rule of [8], from real filling factors to the upper-half complex conductivity plane, and the latter being a similar generalisation of the flux attachment rule of the same reference. This generalisation is natural, and is indeed forced on us if the law of corresponding states is combined with rotational invariance in the bulk of a two-dimensional sample. To see this observe that the law of corresponding states is formulated in terms of a discrete set of transformations on quantum Hall states labelled by $\nu$, or $\sigma_{x y}, \sigma_{x y} \rightarrow \sigma_{x y}+1$ and $\sigma_{x y} \rightarrow \sigma_{x y} /\left(2 \sigma_{x y}+1\right)$. But $\sigma_{x y}$ is only one component of a tensor, 


$$
\sigma_{i j}=\left(\begin{array}{ll}
\sigma_{x x} & \sigma_{x y} \\
\sigma_{y x} & \sigma_{y y}
\end{array}\right)
$$

Two-dimensional rotational invariance demands that $\sigma_{x x}=\sigma_{y y}$ and $\sigma_{x y}=-\sigma_{y x}$, where the latter is a parity violating effect induced by the external field. It is incompatible with rotational invariance to have a transformation acting on one component of a tensor, $\sigma_{x y}$, but not on the other, $\sigma_{x x}$. This causes no problems on the Hall plateau, where $\sigma_{x x}=0$, but is inconsistent when $\sigma_{x x} \neq 0$. To see how to extend the law of corresponding sates to non-zero $\sigma_{x x}$, in is convenient use complex coordinates in two dimensions,

$$
z=x+i y, \quad \bar{z}=x-i y,
$$

in terms of which

$$
\sigma_{i j}=\left(\begin{array}{cc}
\sigma_{x x} & \sigma_{x y} \\
-\sigma_{x y} & \sigma_{x x}
\end{array}\right) \rightarrow\left(\begin{array}{cc}
0 & \sigma_{x x}+i \sigma_{x y} \\
\sigma_{x x}-i \sigma_{x y} & 0
\end{array}\right)=\left(\begin{array}{cc}
0 & i \bar{\sigma} \\
-i \sigma & 0
\end{array}\right) .
$$

Thus, in complex co-ordinates, the tensor $\sigma_{i j}$ is described by $\sigma=\sigma_{x y}+i \sigma_{x x}$ (with $\sigma_{x x} \geqslant 0$ and its complex conjugate. The extension of the law of corresponding states to this complex conductivity leads naturally to the group $\Gamma_{0}(2)$. It is noteworthy that the law of corresponding states [8] was introduced in the quantum Hall effect at almost exactly the same time as the group $\Gamma_{0}(2)$ [2], though from completely different motivations-both sets of authors appear to have been unaware of each other's work.

Because the group action is assumed to commute with the RG flow the critical exponents near a critical point $\sigma_{c}$ are identical with those of $\gamma\left(\sigma_{c}\right)$, in accord with the universality hypothesis of [13]. A flow diagram compatible with $\Gamma_{0}(2)$ is shown in Fig. 1-such diagrams were first produced in Refs. [2,5] and their similarity to the diagrams proposed in Refs. [13,15], without any use of $\Gamma_{0}(2)$ but using more specific reasoning, is manifest.

Under the action of $\Gamma_{0}(2)$ the $\beta$-function, (11), necessarily transforms as

$$
\beta(\gamma(\sigma), \gamma(\bar{\sigma}))=\frac{d}{d s}\left(\frac{a \sigma+b}{2 c \sigma+d}\right)=\frac{1}{(2 c \sigma+d)^{2}} \beta(\sigma, \bar{\sigma})
$$

since $a d-2 b c=1$. It is thus very tempting to assume that $\beta$ is a complex analytic function of $\sigma$ on the upper-half complex plane as such functions, called modular functions of weight -2 , have been extensively studied in the mathematical literature and have many remarkable properties [21]. ${ }^{2}$ A justification of this assumption from first principles, using a microscopic Hamiltonian with specific interactions, will not be attempted here. Rather the consequences of the analyticity assumption will be examined and it will be shown that it gives rise to crossovers that are remarkably similar to the experimental data-thus giving impetus to the more difficult problem of finding a microscopic explanation of analyticity. The present motivation for this assumption really comes from the unexpected direction of supersymmetric Yang-Mills theory, where the group $\Gamma_{0}(2)$ also plays a fundamental rôle and the existence of holomorphic $\beta$-functions gives rise to a

\footnotetext{
${ }^{2}$ More accurately a modular form is meromorphic in $q=\mathrm{e}^{i \pi \sigma}$ rather than $\sigma$ itself.
} 
beautifully rich structure. We shall adapt some of the ideas used in [10], in applying $\Gamma_{0}(2)$ symmetry to the RG flow of supersymmetric Yang-Mills theory, based on the seminal work of Seiberg and Witten [18]. Analyticity means that (13) reads

$$
\beta(\gamma(\sigma))=\frac{d}{d s}\left(\frac{a \sigma+b}{2 c \sigma+d}\right)=\frac{1}{(2 c \sigma+d)^{2}} \beta(\sigma) .
$$

The requirement that $\Gamma_{0}(2)$ commutes with the RG flow means that, at a critical point $\sigma_{c}, \beta\left(\gamma\left(\sigma_{c}\right)\right)=\beta\left(\sigma_{c}\right)$ [6] (this is a generalisation to the non-abelian group $\Gamma_{0}(2)$ of a similar condition for the compatibility of the $\mathbb{Z}_{2}$ Kramers-Wannier type duality with RG flow, analysed in [22]). This can happen if $\beta\left(\sigma_{c}\right)=0$, but also if $\beta\left(\sigma_{c}\right)=\infty$. We shall see that the latter possibility is of interest for crossover in the $\mathrm{QH}$ effect.

There is a theorem ( (4.3.4) in [21]) that any function obeying (14) and satisfying certain reasonable meromorphicity properties must have a special form. Let

$$
f(\sigma)=-\frac{\vartheta_{3}^{4} \vartheta_{4}^{4}}{\vartheta_{2}^{8}}
$$

where $\vartheta_{i}, i=1,2,3$ are Jacobi $\vartheta$-functions (see Appendix A for details). Then $f(\sigma)$ is invariant under $\Gamma_{0}(2)$ (i.e. $f(\gamma(\sigma)=f(\sigma)$ ) and any function obeying (14) must be of the form

$$
\beta(\sigma)=\frac{P(f)}{f^{\prime} Q(f)},
$$

where $P$ and $Q$ are polynomials in $f(\sigma)$.

In the next section, Eq. (16) will be taken as a starting point and plausible explicit analytic expressions for $\sigma(s)$ will be derived, using some further simplifying assumptions.

\section{Renormalisation group flow}

The postulated form of the $\beta$-function, (16), can be restricted even more by making some further assumptions. Let $P(f)=c \prod_{i=1}^{N}\left(f-a_{i}\right)$ be a polynomial of order $N$ and $Q(f)=\prod_{j=1}^{M}\left(f-b_{j}\right)$ be a polynomial of order $M$, where $c, a_{i}$ and $b_{j}$ are constants, with no $a_{i}$ equal to any $b_{j}$ so that there are no common factors between the two polynomials. Then $\beta$ vanishes for any $\sigma_{i}$ such that $f\left(\sigma_{i}\right)=a_{i}$ and diverges for any $\sigma_{j}$ such that $f\left(\sigma_{j}\right)=b_{j}$, where $f(\sigma)$ is defined in (15). From the theory of modular forms [21] it is known that only the fundamental domain of $\Gamma_{0}(2)$ (see Fig. 2 and Appendix A) need be considered as all other values of $\sigma$ in the upper-half complex plane can be reached by acting on the fundamental domain by some element, $\gamma$, of $\Gamma_{0}(2)$. Any critical point of $\beta(\sigma)$ in the fundamental domain has an image in every copy of the fundamental domain under the action of $\Gamma_{0}(2)$. It is a property of the theory of modular forms that $f(\sigma)$ takes all possible complex values at least once in the fundamental domain, and hence in all copies of the domain. 
As explained in the previous section, the fixed points of $\Gamma_{0}(2)$ must be critical points of the RG flow ( $\sigma_{c}$ is a fixed point of $\Gamma_{0}(2)$ if there exists a non-trivial element $\gamma \in \Gamma_{0}(2)$ such that $\left.\gamma\left(\sigma_{c}\right)=\sigma_{c}\right)$. The only fixed points of $\Gamma_{0}(2)$ in the fundamental domain are $\sigma_{c}=0,1, \frac{1+i}{2}$ and the point at infinity, $i \infty$. These four points, and all their images under $\Gamma_{0}(2)$, must be fixed points of the RG flow. That $\sigma_{c}=\frac{n+i}{2}$, for integral $n$, is a critical point has substantial experimental and theoretical foundation $[23,24]$. Of course $\sigma=1$ is the Hall plateau with filling factor $\nu=1$ while $\sigma=0$ is the insulating phase, both of which are expected to be attractive fixed points. The point $\sigma=i \infty$ would correspond to a super-conducting phase, but this is not accessible experimentally, although it is the point about which perturbation theory is performed in field theory calculations (see e.g. the article by Pruisken in [17]). There is no experimental evidence for any new critical points beyond the insulating phase $\sigma=0$, the stable Hall plateaux (all of which are obtained from $\sigma=1$ under the action of $\Gamma_{0}(2)$ ) and the critical point $\sigma_{c}=\frac{1+i}{2}$, and its images under $\Gamma_{0}(2)$. Experiment therefore indicates that there are no new critical points in the fundamental domain beyond those already mentioned at $0,1, \frac{1+i}{2}$ and $i \infty$. This means that the only allowed values of $a_{i}$ and $b_{j}$ in $P(f)$ and $Q(f)$ are $f(0), f(1), f\left(\frac{1+i}{2}\right)$ and $f(i \infty)$-their images under the action of any $\gamma \in \Gamma_{0}(2)$ have the same values of $f$ since $f$ is invariant by construction. In fact $f(0)=f(1)=0, f(i \infty)=-\infty$ and $f\left(\frac{1+i}{2}\right)=\frac{1}{4}$ ( see Appendix A). Obviously one does not take any $a_{i}$ or $b_{j}$ to be infinite, so one is led to the form

$$
\beta=c \frac{f^{n}\left(f-\frac{1}{4}\right)^{m}}{f^{\prime}},
$$

where $m$ and $n$ are integers.

It can be proven, using standard properties of Jacobi $\vartheta$-functions (see Eq. (A.9)) that

$$
f^{\prime}=-i \pi\left(\theta_{3}^{4}+\theta_{4}^{4}\right) f
$$

Thus Eq. (17) can be re-expressed as

$$
\beta(\sigma)=\frac{i c}{\pi} \frac{f^{n-1}\left(f-\frac{1}{4}\right)^{m}}{\left(\theta_{3}^{4}+\theta_{4}^{4}\right)} .
$$

The integers $n$ and $m$ can be constrained by examining various limits. The stability of the Hall plateau at $\sigma=1$, where $f(1)=0$, demands that $\beta=0$ when $f=0$. The points $\sigma=1$ and $\sigma=0$ are mapped onto each other under the action of $\Gamma_{0}(2)$, so $\beta$ also vanishes at $\sigma=0$, where $f(0)=0$ also. Since $\vartheta_{3}^{4} \approx-1 / \sigma^{2}, \vartheta_{4} \rightarrow 0$ and $f \approx-16 e^{-i \pi / \sigma}$ as $\sigma \rightarrow 0$, (A.11), it is necessary that $n-1 \geqslant 0$.

If the assumptions being made here have any validity at all, the limit $\sigma \rightarrow i \infty$ should have some affinity with the weak coupling limit of the non-linear $\sigma$-model approach to the QH effect, advocated by Pruisken in [17]. Quantitative comparison of the form proposed here with Pruisken's asymptotic form is hampered by the fact that the functional form of $l(\tilde{b})$ is not known in general-indeed the assumption that the 
$\beta$ function is analytic is presumably only true, if it is true at all, in a restricted class of renormalisation schemes. In fact the form of the $\beta$-functions suggested here implies that they cannot be the same $\beta$-functions of Pruisken's analysis in [17] as the latter are definitely not holomorphic as $\sigma_{x x} \rightarrow \infty$, as noted in [9] (see comments in the last paragraph of Section 5). Nevertheless it may be reasonable to extract some gross features from the weak coupling analysis. In particular we shall assume that for $\sigma_{x x}$ large:
(i) $\quad \beta$ is finite;
(ii) $\sigma_{x y}$ is a constant, i.e. $\beta_{x y}=0$;
(iii) $\beta_{x x} \leqslant 0$.

It is to be expected that these three conditions are rather general, regardless of whether or not holomorphicity holds.

Now $f \rightarrow-\infty$ and $\vartheta_{3}^{4}=\vartheta_{4}^{4}=1$ as $\sigma \rightarrow i \infty$, so (i) above requires that $n+m-1 \leqslant 0$. Since $n \geqslant 1$ we thus have $m \leqslant 0$. In perturbation theory condition (ii) comes from the assumption that instanton effects are negligible in the weak coupling limit and therefore $\sigma_{x y}$, which is a topological parameter in the field theory model of Pruisken [17], does not run in this limit. Using the asymptotic form (A.10) for large $\sigma_{x x}$ in Eq. (19) gives

$$
\beta=\frac{c}{2 \pi}\left(\frac{-e^{2 \pi \sigma x x}}{256}\right)^{n+m-1}\left[\sin \left\{2 \pi(n+m-1) \sigma_{x y}\right\}+i \cos \left\{2 \pi(n+m-1) \sigma_{x y}\right\}\right] .
$$

Condition (ii) above then demands that $n=-m+1$. Finally condition (iii) requires that $c$ be real and negative. Without loss of generality $c=-1$, by rescaling $s$ if necessary, and one is finally led to the form

$$
\beta(\sigma)=-\frac{1}{f^{\prime}} \frac{f^{n}}{\left(f-\frac{1}{4}\right)^{n-1}}=-\frac{i}{\pi} \frac{1}{\left(\vartheta_{3}^{4}+\vartheta_{4}^{4}\right)}\left(\frac{f}{f-\frac{1}{4}}\right)^{n-1},
$$

with $n \geqslant 1$. The function (22) with $n=0$ is actually the form relevant to $N=2$ super-symmetric Yang-Mills theory.

Eq. (22) can now be integrated exactly along any single trajectory. Writing it as

$$
\frac{d s}{d \sigma}=-\frac{d f}{d \sigma} \frac{1}{f^{n}} \sum_{r=0}^{n-1}\left(\begin{array}{c}
n-1 \\
r
\end{array}\right)\left(-\frac{1}{4}\right)^{n-1-r} f^{r}
$$

this integrates to

$$
s(\sigma)=-\ln \left(\frac{f}{f_{0}}\right)-\sum_{r=0}^{n-2}\left(\frac{1}{r-n+1}\right)\left(\begin{array}{c}
n-1 \\
r
\end{array}\right)\left(-\frac{1}{4}\right)^{n-1-r}\left(f^{r-n+1}-f_{0}^{r-n+1}\right),
$$


where $f_{0}=f(\sigma(s=0))$, which can be inverted to give $\sigma(s)$. For crossover between $\nu=0$ and $\nu=1$ we take $f_{0}=\frac{1}{4}$ with $s\left(\frac{1+i}{2}\right)=0$. Using the limiting form (A.11) for $f(\sigma)$ as $\sigma \rightarrow 0, f \approx-16 e^{-i \pi / \sigma}$, there is a quantitative difference between $n=1$ and $n>1$ in the limit of large $s$. In the former case, the sum is absent and only the logarithm survives, giving $\sigma \approx i \pi / s$, while in the latter case the term in the sum with $r=0$ dominates and $s \approx\left(\frac{1}{n-1}\right)\left(\frac{1}{64}\right)^{n-1} e^{i \pi(n-1) / \sigma}$ so $\sigma \approx i \pi(n-1) / \ln s$. The case $n=1$ therefore approaches the stable fixed points at $\sigma=0$ and 1 for large $s$ much faster than for $n>1$. Although $s$ has not yet been determined it is assumed to be a monotonic function of $\Delta B$ so, whatever its functional form, $n=1$ will give faster convergence to Hall plateaux than $n>1$. Experimentally, the former possibility is to be preferred over the latter.

To recapitulate, Eq. (22) is the most general form of the $\beta$-function compatible with the following criterion:

(i) Stability of Hall plateaux.

(ii) Gross features of weak coupling in the asymptotic limit of the field theoretical models.

(iii) No new critical points of the RG flow other than the insulating phase, Hall plateaux, critical points in the crossover between Hall plateaux and the weak coupling limit.

(iv) Symmetry of $\mathrm{QH}$ states under the action of $\Gamma_{0}(2)$ on the upper-half complex conductivity plane which commutes with the RG flow.

(v) Complex analyticity of the $\beta$-functions.

Finally the case $n=1$ gives the fastest approach to the stable fixed points corresponding to Hall plateaux. The RG flow for the case $n=1$ was considered in [11] within the context of Yang-Mills gauge theory. It is reproduced in Fig. 1 here, which was obtained by observing that integrating (22) with $n=1$ using an arbitrary integration constant gives

$$
s-s_{0}=-\ln \left(\frac{f}{f_{0}}\right),
$$

where $f_{0}=f\left(s_{0}\right)$. Now $s$ is a real parameter, therefore $\arg (f)=\arg \left(f_{0}\right)$, and the phase of the function $f$ in Eq. (15) is constant along RG trajectories. Fig. 1 is simply a contour plot of the complex phase of $f(\sigma)$, and it reproduces the flows predicted in Refs. $[13,15]$. Note that $\sigma_{c}=\frac{1+i}{2}$ is a repulsive fixed point. For most of the following the case $n=1$ will be assumed, except where explicitly stated.

\section{The critical point at $\sigma_{c}=\frac{1+i}{2}$}

The element

$$
\gamma=\left(\begin{array}{ll}
1 & -1 \\
2 & -1
\end{array}\right)
$$


of $\Gamma_{0}(2)$ leaves the point $\sigma_{c}=\frac{1+i}{2}$ fixed and so $\sigma_{c}$ must be a critical point of the RG flow, by the arguments of Section 3. $\sigma_{c}$ therefore corresponds to the critical point in the crossover from $\sigma=0$ to $\sigma=1$. Numerical calculations, based on models for the microscopic physics, support this conclusion [24]. It is shown in Appendix A that $\vartheta_{3}^{4}=-\vartheta_{4}^{4}$ and $f=\frac{1}{4}$ at $\sigma=\sigma_{c}=\frac{1+i}{2}$. Since $n \geqslant 1$ it is immediately apparent from (22) that $\beta\left(\sigma_{c}\right)$ diverges. This is a critical point that is characterised not by a vanishing $\beta$-function but by a singular one. However, the situation is not as bad as it might seem at first sight. For $\sigma$ close to the critical point, $\sigma=\sigma_{c}+\varepsilon$ with $\varepsilon$ small,

$$
f\left(\sigma_{c}+\varepsilon\right)=\frac{1}{4}-\frac{\{\Gamma(1 / 4)\}^{8}}{64 \pi^{4}} \varepsilon^{2}+o\left(\varepsilon^{4}\right),
$$

so

$$
\left.f^{\prime}\right|_{\sigma_{c}}=-\frac{\{\Gamma(1 / 4)\}^{8}}{32 \pi^{4}} \varepsilon+o\left(\varepsilon^{3}\right),
$$

see (A.14) $(\Gamma(1 / 4)$ here is the usual Gamma function). So the mildest form of singularity is

$$
\frac{d \varepsilon}{d s} \approx \frac{8 \pi^{4}}{\{\Gamma(1 / 4)\}^{8} \varepsilon}
$$

for $n=1$. This integrates to $\varepsilon^{2} \approx 16 \pi^{4} s /\{\Gamma(1 / 4)\}^{8}$, where the zero of $s$ has been chosen so that $\sigma(s=0)=\sigma_{c}$. Thus $\varepsilon$ is either purely real or purely imaginary, depending on whether $s>0$ or $s<0$. Any other value is repulsed from $\sigma_{c}$, as it is an unstable fixed point.

For $\varepsilon$ real the choice $n=1$ thus leads to the form $\varepsilon \propto \sqrt{s}, \operatorname{Re}(\beta) \propto 1 / \sqrt{s}$ and $\operatorname{Im}(\beta) \approx 0$, with $\sigma(s)$ itself finite at $s=0$. Only the slope of $\sigma_{x y}$ is singular at the critical point. In fact a glance at the experimental data for crossover between any two $\mathrm{QH}$ states with $\sigma_{x y}=\nu_{1}$ and $\sigma_{x y}=\nu_{2}$ makes it clear that one expects the slope of $\sigma_{X Y}(B)$ to be large (and indeed infinite as the temperature $T \rightarrow 0$, where one expects a step function). Thus this singularity is not necessarily a disaster. For finite temperatures, the infinite slope can be avoided by a suitable choice of $s(\Delta B)$ or, equivalently, $s(\Delta \nu)$. Recall that $s(\Delta B)$ is taken to be a monotonic function of the external magnetic field $B$ and one is free to choose the zero of $s$ so that $s(\Delta B=0)=0$, or $s(\Delta \nu=0)=0$. We assume that $s(\Delta \nu)$ is a real analytic function of $\Delta \nu$ at $\Delta \nu=0$, otherwise the definition of the $\beta$-function in (11) hardly makes sense. ${ }^{3}$ Experimentally [12] $\sigma_{x x}$ and $\sigma_{x y}$ are perfectly well behaved functions of $\Delta \nu$ near $\Delta \nu=0$, they look analytic with $d \sigma_{x y} / d(\Delta \nu)>0$ and finite, and $d \sigma_{x x} / d(\Delta \nu)=0$. If we therefore assume a Taylor expansion

$$
\sigma_{x y}(\Delta \nu)=\frac{1}{2}+C \Delta \nu+\ldots
$$

\footnotetext{
${ }^{3}$ I thank Denjoe O'Connor for discussions on this point.
} 
with $C$ a positive constant, we see that $\Delta \nu \propto \varepsilon \propto \sqrt{s}$, i.e. $s \propto(\Delta \nu)^{2}$. This gives rise to a singularity in $d \sigma_{x y} / d s$ at $s=0$, even though $d \sigma_{x y} / d(\Delta \nu)$ is perfectly finite for finite temperature. This is the source of the pole in the $\beta$-function at $\sigma_{c}=\frac{1+i}{2}$.

For general $n \geqslant 1$ a similar analysis shows that $d \epsilon / d s \propto 1 / \epsilon^{2 n-1}$ near the critical point, giving $\epsilon \propto s^{1 /(2 n)}$ and $\operatorname{Re}(\beta) \propto s^{-((2 n-1) / 2 n)}$. Analyticity of $s(\Delta \nu)$ at the critical point then requires $s \approx(\Delta \nu)^{2 n}$. From now on only $n=1$ will be considered as it appears likely to be the most relevant in order to achieve the most rapid approach to the fixed points on the real axis.

It may be worthwhile re-iterating this point, as it is at first sight surprising that criticality is associated with an infinite $\beta$-function, when one normally associates a vanishing $\beta$-function with a critical point. There is no unique definition of the $\beta$ function-there are many ways of defining it, all of which are physically equivalent of course. Thus $\beta_{L}:=L d \sigma / d L$ is not the same as $\beta_{\Delta \nu}:=d \sigma / d \Delta \nu$ ( $L$ here is the same as in Section 2). Indeed $\beta_{\Delta \nu}$ is non-zero at the critical point (and finite for $T>0$ ) whereas $\beta_{L}$ vanishes there, and $(1 / \Delta \nu) d \sigma / d \Delta \nu$ diverges at the critical point, where $\Delta \nu=0$. It is $(1 / \Delta \nu) d \sigma / d \Delta \nu$ which is obtained from the analytic $\beta$-function proposed here. $\beta_{L}$ and $\beta_{\Delta y}$ give perfectly good descriptions of the physics - but they will not be complex analytic, in general. Note that it is $\beta_{L}$ which is accessed by the field theoretical analysis in [17].

\section{Analytic form of the crossover}

In this section we consider the explicit analytic form of the function $\sigma(s)$, for $n=1$, in crossing over from $\sigma=0$ to $\sigma=1$ along the semi-circular arch of unit diameter passing through $\sigma=\frac{1+i}{2}$. All QH transitions, between $\sigma=\nu_{1}=p_{1} / q_{1}$ and $\sigma=\nu_{2}=p_{2} / q_{2}$ with $p_{2} q_{1}-p_{1} q_{2}=1$, can be obtained by mapping this semi-circle onto the semi-circle bridging $\nu_{1}$ and $\nu_{2}$, with diameter $1 / q_{1} q_{2}$ passing through, $\gamma\left(\sigma_{c}\right)=$ $\left(p_{1} q_{1}+p_{2} q_{2}+i\right) /\left(q_{1}^{2}+q_{2}^{2}\right)$. We have, from (25),

$$
f(\sigma)=f_{0} \mathrm{e}^{-\left(s-s_{0}\right)} .
$$

Choosing the zero of $s, s_{0}$, so that $s=0$ corresponds to the point $\gamma\left(\sigma_{c}\right)$, where $f\left(\gamma\left(\sigma_{c}\right)\right)=f_{0}=1 / 4$ results in

$$
-\frac{\vartheta_{3}^{4} \vartheta_{4}^{4}}{\vartheta_{2}^{8}}=f(\sigma)=\frac{1}{4} \mathrm{e}^{-s}
$$

Thus the semi-circular arches giving crossover between any two $\mathrm{QH}$ states are characterised by $\arg (f)=0$. The explicit functional form of $\sigma(s)$ can now be determined in terms of complete elliptic functions of the second kind,

$$
K(k)=\int_{0}^{\pi / 2} \frac{d \varphi}{\sqrt{1-k^{2} \sin ^{2} \varphi}},
$$


using standard formulae [25] (see Appendix A),

$$
\vartheta_{2}=\sqrt{\frac{2 k K(k)}{\pi}}, \quad \vartheta_{3}=\sqrt{\frac{2 K(k)}{\pi}}, \quad \vartheta_{4}=\sqrt{\frac{2 k^{\prime} K(k)}{\pi}},
$$

where the modulus, $k$, is related to $\sigma$ by $\mathrm{e}^{i \pi \sigma}=\mathrm{e}^{-\pi K^{\prime} / K}$, with $K^{\prime}(k)=K\left(k^{\prime}\right)$ and $\left(k^{\prime}\right)^{2}=1-k^{2}$ the complementary modulus. Thus

$$
\sigma=2 \bar{m}+\frac{i K^{\prime}}{K}
$$

for integral $\bar{m}$ (for convenience the relevant formulae are reproduced in Appendix A). The integer $\bar{m}$ can be set to zero, since the other possibilities follow from repeated application of the symmetry $\sigma \rightarrow \sigma+1$ of $\Gamma_{0}(2)$. Using the relations (33) in (31) gives $s$ in terms of $k$,

$$
\frac{1}{4} \mathrm{e}^{-s}=-\frac{\vartheta_{3}^{4} \vartheta_{4}^{4}}{\vartheta_{2}^{8}}=-\frac{\left(k^{\prime}\right)^{2}}{k^{4}} \Rightarrow k^{2}=2 \mathrm{e}^{s}\left(1 \pm \sqrt{1-e^{-s}}\right) .
$$

For $0 \leqslant s<\infty$ the plus sign gives $2 \leqslant k^{2}<\infty$ and the negative sign gives $1<k^{2} \leqslant$ 2 -the two branches combined give the range $1<k^{2}<\infty$, and so $-\infty<\left(k^{\prime}\right)^{2}<0$ (it has already been shown-see the discussion after Eq. (28)-that $s<0$ corresponds to passing through $\sigma_{c}$ in the purely imaginary direction, which is not relevant to crossover). Since $K(k)$ is complex in this range it is convenient to manipulate Eq. (34), using some identities between elliptic integrals given in Appendix A, in order to expose the real and imaginary parts of (34) explicitly. First note that $w^{2}:=1 / k^{2}$ has the range $0<w^{2}<1$, so Eq. (A.19) allows one to write

$$
K(k)=w\left\{K(w)+i K^{\prime}(w)\right\},
$$

with $K(w)$ and $K^{\prime}(w)$ real. Next define a real variable $u$ via $k^{\prime}=i u$, with $0<u<\infty$, $w^{2}=1 /\left(1+u^{2}\right)$ and $\left(w^{\prime}\right)^{2}=1-w^{2}=u^{2} /\left(1+u^{2}\right)$. Then Eq. (A.20) with $k$ replaced by $w^{\prime}$, gives

$$
K^{\prime}(k)=K(i u)=w K^{\prime}(w) .
$$

Using Eqs. (36) and (37) in (34), with $\vec{m}=0$, results in

$$
\sigma(s)=\frac{K^{\prime}(w)\left\{K^{\prime}(w)+i K(w)\right\}}{\left[\{\bar{K}(w)\}^{2}+\left\{K^{\prime}(w)\right\}^{2}\right]}
$$

which gives an explicit analytic form for $\sigma(s)$ in which the real and imaginary parts are manifest, since

$$
w=\frac{1}{k}=\sqrt{\frac{1 \mp \sqrt{1-e^{-s}}}{2}}
$$

from (35), lies in the range $0<w<1$. 
In order to show that the above equation does indeed describe crossover between $\sigma=0$ and $\sigma=1$ we shall analyse various limits of (38). For $s=0$ both branches give $w^{2}=\frac{1}{2}$ and so $\sigma(0)=\frac{1+i}{2}$, since $K(1 / \sqrt{2})=K^{\prime}(1 / \sqrt{2})$, thus $s=0$ is indeed the critical point. As $w \rightarrow 0$ ( $s \rightarrow \infty$ on the upper branch), the asymptotic forms of the complete elliptic integrals (Eqs. (A.17) and (A.18)) can be used to write

$$
\begin{aligned}
& K\left(w^{\prime}\right)=\frac{\pi}{2}+o\left(w^{2}\right)=\frac{\pi}{2}+o\left(\mathrm{e}^{-s}\right), \\
& K^{\prime}(w)=\ln \left(\frac{4}{w}\right)+o\left(w^{2} \ln w\right)=\frac{s}{2}+\ln 8+o\left(s \mathrm{e}^{-s}\right),
\end{aligned}
$$

so

$$
\sigma=\frac{(s+6 \ln 2)(s+6 \ln 2+i \pi)}{\left\{\pi^{2}+(s+6 \ln 2)^{2}\right\}}+o\left(s \mathrm{e}^{-s}\right) \rightarrow 1 .
$$

As $w \rightarrow 1\left(s \rightarrow \infty\right.$ on the lower branch), the rôles of $w$ and $w^{\prime}$ are interchanged and

$$
\sigma=\frac{\pi\{\pi+i(s+6 \ln 2)\}}{\left\{\pi^{2}+(s+6 \ln 2)^{2}\right\}}+o\left(s \mathrm{e}^{-s}\right) \rightarrow 0 .
$$

Thus the two branches combined, with $0<w<1$, do indeed interpolate between $\sigma=0$ and $\sigma=1$ with $w=1 / \sqrt{2}$ giving the critical point at $\sigma_{c}=\frac{1+i}{2}$.

To compare this analytic expression for $\sigma(s)$ with the available experimental data, we must still address the question of how the variable $s$ is depends on the external magnetic field, or equivalently on $v=\left(\bar{n} e / T^{\mu}\right) \Delta \nu$. One piece of information that has not yet been brought into play, and can be used to constrain the form of $s$, is the particle-hole transformation rule of [8]. This implies a symmetry under $\nu \rightarrow 1-\nu$, or $\Delta \nu \rightarrow-\Delta \nu$, which suggests that $s$ should be an even function of $\Delta \nu .{ }^{4}$ This is compatible with the mathematical analysis in the previous section, where it was shown that the apparent regularity in the experimental data of $\sigma_{x y}$ at the critical point implies that $s \propto v^{2}$, at least for $\Delta \nu$ small. As a first try, therefore, we shall take $s=\left(A \Delta \nu / T^{\mu}\right)^{2}$, for some real positive constant $A$. It may be significant that the experimental value of the critical exponent in $(6), \nu_{\xi} \approx 2.02$, is so close to two. If it were precisely two, then $s$ would be proportional to the inverse correlation length, $s \propto 1 / \xi$. It would also be quite natural if $\mu$ were exactly one half, giving $s \propto 1 / T$. The experimental data, however, seem to indicate a somewhat lower value for $\mu$ (and correspondingly a higher value for $\nu_{\xi}$ ).

Using $s=\left(A \Delta \nu / T^{\mu}\right)^{2}$ and the four values for the temperature quoted for Fig. $2 \mathrm{~b}$ in [12], $T=42,84,106$ and $145 \mathrm{mK}$, with the best fit experimental value of $\mu=$ $0.45 \pm 0.05$, the functions

$$
\begin{aligned}
& \sigma_{x y}(s)=\frac{\left\{K^{\prime}(w)\right\}^{2}}{\left[\left\{K^{\prime}(w)\right\}^{2}+\{K(w)\}^{2}\right]}, \\
& \sigma_{x x}(s)=\frac{K^{\prime}(w) K(w)}{\left[\left\{K^{\prime}(w)\right\}^{2}+\{K(w)\}^{2}\right]},
\end{aligned}
$$

\footnotetext{
${ }^{4}$ We shall see later that this is strictly true only for integer transitions.
} 
can be plotted, with

$$
w=\sqrt{\frac{1-\operatorname{sign}(\Delta \nu) \sqrt{1-\mathrm{e}^{-\left(A \Delta \nu / T^{\mu}\right)^{2}}}}{2}},
$$

for the transition $\nu: 0 \rightarrow 1$, and the result for $\rho_{x x}$ and $\rho_{x y}$ are shown in Fig. 3, where the choices $A=60, \mu=0.50$ have been made, since these seem to give a good visual fit to the experimental data in Fig. $2 b$ of [12]. A better fit to the data is obtained if $\rho_{x x}$ is rescaled by a constant, so as to raise the critical value of $\rho_{x x}$ above the predicted value of unity - the experimental curve in [12] gives a value greater than one whereas $\Gamma_{0}(2)$ symmetry predicts exactly one. If the assumptions made here are correct, this is presumably due to the experimental difficulties involved in determining $\rho_{x x}$ (see for example the comments in Cage's article in [17]). Note that a prediction here is that, for this transition, $\rho_{x y}=1$ well into the insulating phase-a fact that is well borne out by experiment. Similarly, with the four values $T=42,70,101$ and $137 \mathrm{mK}$ used in Fig. 1 of [12] the functions

$$
\begin{aligned}
& \sigma_{x y}(s)=1+\frac{\left\{K^{\prime}(w)\right\}^{2}}{\left[\left\{K^{\prime}(w)\right\}^{2}+\{K(w)\}^{2}\right]}, \\
& \sigma_{x x}(s)=\frac{\left.K^{\prime}(w) K(w)\right\}}{\left[\left\{K^{\prime}(w)\right\}^{2}+\{K(w)\}^{2}\right]},
\end{aligned}
$$

for the transition $\nu: 1 \rightarrow 2$, are plotted in Fig. 4, together with the corresponding resistivities. Fig. 4 was produced with the choice $A=40, \mu=0.50$, again with a view to a good visual fit to the experimental data in Fig. 1 of Ref. [12], and again the fit for $\rho_{x y}$ is noticeably better than that for $\rho_{x x}$.

It is stressed that there are only two parameters which can be varied in the analytic expressions to produce Figs. 3 and 4 , the scaling exponent $\mu$ and the constant $A-\mu$ is assumed to be universal and is taken from experiments, which then leaves only one parameter, $A$, which can be varied in order to fit the experimental data. In general $A$ is not expected to be universal--it would depend on various parameters such as the electron and impurity density. In particular it depends on the critical magnetic field and so is different for each transition.

It should also be borne in mind that the form (45) for $w(\Delta \nu)$ could have higher corrections in $\Delta \nu$, it is only for $\Delta \nu$ near zero that we can trust $s \propto\left(\Delta \nu / T^{\mu}\right)^{2}$. However, assuming that $s$ is an even function of $\Delta \nu$, as the particle-hole transformation mentioned earlier suggests, one expects the corrections to be of order $(\Delta \nu)^{4}$. In the experimental data from Ref. [12], the range of $\Delta \nu$ required for crossover is $<0.1$ so corrections to the leading $(\Delta \nu)^{2}$ term can be expected to be less than $1 \%$, unless something conspires to produce large coefficients.

The particle-hole transformation extends to complex $\sigma$ as $\sigma \rightarrow 1-\bar{\sigma}$, as is evident from Fig. 2, (this is not an element of $\Gamma_{0}(2)$, but rather an outer auto-morphism of that group). Thus it is to be expected that the longitudinal conductivity, $\operatorname{Im}\{\sigma(\Delta \nu)\}$, is an even function of $\Delta \nu$, at least for integral transitions. This property is manifest in 
Eqs. (44) and (46) since, as is easily proven from (45), $w(-\Delta \nu)=w^{\prime}(\Delta \nu)$ where $\left(w^{\prime}\right)^{2}=1-w^{2}$.

This behaviour of $w(\Delta \nu)$ under $\Delta \nu \rightarrow-\Delta \nu$ has an interesting consequence for the resistivity, $\rho=-1 / \sigma$, derived from Eq. (44). The longitudinal resistivity for the transition $\nu: 0 \rightarrow 1$ is

$$
\rho_{x x}=\frac{K(w)}{K^{\prime}(w)} .
$$

Thus we have

$$
\rho_{x x}(-\Delta \nu)=\frac{1}{\rho_{x x}(\Delta \nu)},
$$

for $\nu: 0 \rightarrow 1$, which is well supported experimentally [14,26,27]. In particular in Ref. [26] the authors fit the form $\rho_{x x}(\Delta \nu)=\mathrm{e}^{-\Delta \nu / \tilde{\nu}(T)}$ to the data, where $\tilde{\nu}(T)$ is a function of $T$. They find that $\tilde{\nu}(T)=T^{\mu}$ is incompatible with the data for any $\mu$ and suggest instead that a linear form, $\tilde{\nu}(T)=\tilde{\alpha} T+\tilde{\beta}$ with $\tilde{\alpha}$ and $\tilde{\beta}$ non-zero constants, gives a better fit and this is interpreted as a violation of the scaling hypothesis. It would be very interesting to check whether or not the alternative form (47) is compatible with the experimental data because, if it is, then the experiments in $[14,26,27]$ would provide confirmation of scaling and not a violation of it.

Taking (38) as a template, the assumption of $\Gamma_{0}(2)$ symmetry (which implies universality) allows any transition to be modeled. The crossover $\nu: p_{1} / q_{1} \rightarrow p_{2} / q_{2}$ is obtained from the template transition $\nu: 0 \rightarrow 1$ by the action of

$$
\gamma=\left(\begin{array}{cc}
p_{2}-p_{1} & p_{1} \\
q_{2}-q_{1} & q_{1}
\end{array}\right)
$$

as (see Appendix A)

$$
\sigma \rightarrow \frac{\left(p_{2}-p_{1}\right) \sigma+p_{1}}{\left(q_{2}-q_{1}\right) \sigma+q_{1}}
$$

Using (38) this gives

$$
\sigma(\Delta \nu)=\frac{p_{2} q_{2}\left\{K^{\prime}(w)\right\}^{2}+p_{1} q_{1}\{K(w)\}^{2}+i K^{\prime}(w) K(w)}{\left[\left\{q_{1}^{2} K(w)\right\}^{2}+q_{2}^{2}\left\{K^{\prime}(w)\right\}^{2}\right]}
$$

The form of $w$ as a function of $\Delta \nu$ changes as well, because $\Delta \nu$ for the transition $\nu: p_{1} / q_{1} \rightarrow p_{2} / q_{2}$ is not the same as $\Delta \nu$ for the template transition $\nu: 0 \rightarrow 1$, in general. Denoting the filling factor for the template transition by $\nu_{01}$ (which is the argument of the function $s\left(\Delta \nu_{01}\right)$ ), we expect $\nu_{01}$ to be obtained from $\nu$ using

$$
\gamma^{-1}=\left(\begin{array}{cc}
q_{1} & -p_{1} \\
-\left(q_{2}-q_{1}\right) & p_{2}-p_{1}
\end{array}\right)
$$

so

$$
\nu_{01}=\frac{q_{1} \nu-p_{1}}{\left(q_{1}-q_{2}\right) \nu+\left(p_{2}-p_{1}\right)}
$$


and thus

$$
\Delta \nu_{01}=\frac{\Delta \nu}{\alpha\left\{\left(q_{1}-q_{2}\right) \Delta \nu+\alpha\right\}}:=\frac{\Delta \nu}{\zeta(\Delta \nu)},
$$

where $\alpha:=\left(p_{2}-p_{1}\right)-\left(q_{2}-q_{1}\right) \nu_{c}$ and $\zeta(\Delta \nu):=\alpha\left\{\left(q_{1}-q_{2}\right) \Delta \nu+\alpha\right\} . \Delta \nu$ and $\Delta \nu_{0}$ I are equal for integer transitions of the form $p \rightarrow p+1$ (where $p_{2}=p+1, p_{1}=p$ and $q_{1}=q_{2}=1$ ) but not otherwise. Note that $\alpha \rightarrow-\alpha, q_{1} \rightarrow q_{1}$ and $q_{2} \rightarrow q_{2}$ under $\nu \rightarrow 1-\nu$, hence $\Delta \nu_{01} \rightarrow-\Delta \nu_{01}$ and the discussion of the particle-hole transformation rule, in the paragraph after Eq. (43), should now be modified to say that $s$ could have corrections of order $\left(\Delta \nu_{01}\right)^{4}$.

Thus for a general crossover the argument $w$ appearing in (50) is given by

$$
w=\sqrt{\frac{1-\operatorname{sign}(\Delta \nu) \sqrt{1-\mathrm{e}^{-\left|A \Delta \nu / \zeta(\Delta \nu) T^{\mu}\right|^{2}}}}{2} .}
$$

Eq. (50), with the definition (53), is the main result of this paper. In principle there could be corrections of order $(\Delta \nu)^{4}$ to the exponent in Eq. (53), but in practice it appears to give good agreement with experiment as it stands, since the range of $\Delta \nu$ required for crossover is small. The special case (38) is reproduced by setting $p_{1}=0$ and $p_{2}=q_{1}=q_{2}=1$.

The longitudinal resistivity actually takes the form given in (47) for all of the transitions $\nu: 0 \rightarrow 1 / q$, as can be shown using (50) with $p_{1}=0, p_{2}=q_{1}=1$ and $q_{2}=q$. Thus "rho-duality" (48) should be valid for these transitions, but only these. Eq. (48), with $\Delta \nu$ replaced with $\Delta \nu_{01}$ from (52), was tested experimentally in [14] for the case $\nu: 0 \rightarrow 1 / 3$ (for which $p_{1}=0, p_{2}=q_{1}=1$ and $q_{2}=3$ ) and appears to give support for (52), even for values of $\Delta \nu$ as large as 0.1 , while putting $\zeta(\Delta \nu)$ equal to a constant does not give such good a fit to the data, the discrepancy being a $10 \%$ correction. It therefore seems that (53) is valid even for $\Delta \nu$ as large as 0.1 , at least for the temperature range explored in [14], with the corrections being of order $(\Delta \nu)^{4}$ giving a $1 \%$ error which is within the limits of experimental accuracy.

\section{Conclusions}

In this paper an explicit form of the crossover between two $\mathrm{QH}$ plateaux has been derived, the final result being given in (50) and (53). The resulting crossovers are plotted in Figs. 3 and 4 for the cases $p_{1}=0, p_{2}=q_{1}=q_{2}=1$ and $p_{2}=2, p_{1}=q_{1}=$ $q_{2}=1$, corresponding to $\nu: 0 \rightarrow 1$ and $\nu: 1 \rightarrow 2$ respectively. The results agrees remarkably well with the experimental data in [12], at least qualitatively. It would be of interest to check the results quantitatively, using experimental numbers rather than just the graphical data which are available in the literature.

The assumptions are:

(i) The law of corresponding states of [8] should be extended into the upper-half complex conductivity plane, $\sigma=\sigma_{x y}+i \sigma_{x x}$ (in units of $e^{2} / h$ ), as required by 
rotational invariance in the bulk,. This is encoded mathematically into a group action on $\sigma$-specifically the group is $\Gamma_{0}(2)$,

(ii) The action of $\Gamma_{0}(2)$ commutes with RG flow. This implies universality of $\mathrm{QH}$ transitions.

(iii) There are no extra critical points, other than those already indicated by the experimental data and weak coupling expansions, i.e. $\sigma=0,1, \frac{1+i}{2}, i \infty$ and their images under the action of $\Gamma_{0}(2)$, and that the RG flow be compatible with the experimental data and the robust features of the weak coupling expansion enumerated in (20).

(iv) The $\beta$-functions for the RG flow that describes crossover between two $\mathrm{QH}$ plateaux, or between a $\mathrm{QH}$ state and the insulating phase, are complex analytic functions when described in terms of a real variable $s$ that is a monotonic real analytic function of the external magnetic field.

Assumptions (i) and (ii) above have already received a great deal of attention $[2,5,6,8]$. Despite many successes it is still not yet clear whether universality in $\mathrm{QH}$ transitions is a good hypothesis, but time will tell and for the moment it seems promising to investigate the consequences of these assumptions. Assumption (iii) does not seem to require comment, given (i) and (ii). The new ingredient here is (iv) which necessitates analysis and justification. The only justification given here is that this assumption produces an expression which appears to be in remarkable agreement with experimental data on QH crossover. Given this apparent success, it is important to achieve an understanding of how this form could emerge from the underlying physics. There is no obvious reason from the field theory models considered so far, e.g. Pruisken's non-linear $\sigma$-model with a topological term [17] or Chern-Simons effective actions [28], why an analytic $\beta$-function should give good results. It should probably be borne in mind when trying to analyse the hypothesis of complex analyticity using field theoretic techniques that the notion of complex analyticity would be expected to depend on the renormalisation scheme chosen for any calculations-it could not be expected to be true in an arbitrary scheme. It may be significant for analyticity that if the exponent $\nu_{\xi}$ in (6) were exactly two, then the RG parameter $s$ would be inversely proportional to the correlation length.

Finding a microscopic justification promises to be an involved project, but in view of the tantalising similarity between the analytic results presented here and the experimental data it may be one well worth pursuing.

\section{Note added in proof}

After the completion of this manuscript two further papers analysing possible forms for the $\beta$-function, compatible with $\Gamma_{0}(2)$ symmetry, appeared $[29,30]$. 


\section{Acknowledgements}

It is a pleasure to thank Jan Pawlowski for helpful discussions during the early stages of this work and Jason Twamley for help in producing Fig. 1.

This research was sponsored in part by an Alexander von Humboldt foundation fellowship and in part by financial assistance from Baker Consultants Ltd., Ireland, networking specialists (http://www.baker.ie).

\section{Appendix A}

This appendix contains a summary of the properties of the modular group, its subgroup $\Gamma_{0}(2)$, the Jacobi $\vartheta$-functions and complete integrals of the second kind, which are needed in the text.

The modular group, $\Gamma(1)$, can be represented by the set of all $2 \times 2$ matrices with integer entries and determinant one. Thus $g \in \Gamma(1)$ can be written as

$$
g=\left(\begin{array}{ll}
a & b \\
c & d
\end{array}\right) \quad \text { with } a d-b c=1 .
$$

$\Gamma(1)$ is therefore isomorphic to $\operatorname{Sl}(2, \mathbb{Z})$, which in turn is isomorphic to $\operatorname{Sp}(2, \mathbb{Z})$-the group of symplectic $2 \times 2$ matrices with integer entries. Elements of $\Gamma$ (1) have a natural action on the upper-half complex plane, parameterised here by $\sigma \in \mathbb{C}$ with $\operatorname{Im}(\sigma)>0$, given by

$$
g(\sigma)=\frac{a \sigma+b}{c \sigma+d}
$$

It is easy to check, using $a d-b c=1$, that $\operatorname{Im}(g(\sigma))>0$ if $\operatorname{Im}(\sigma)>0$. The group $\Gamma(1)$ is an infinite discrete group and it is generated by two elements, $U: \sigma \rightarrow \sigma+1$ with

$$
g_{U}=\left(\begin{array}{ll}
1 & 1 \\
0 & 1
\end{array}\right)
$$

and $V: \sigma \rightarrow-1 / \sigma$ with

$$
g_{V}=\left(\begin{array}{cc}
0 & 1 \\
-1 & 1
\end{array}\right)
$$

any element can be represented by some combination of products of such matrices (though not necessarily uniquely). The group $\Gamma(1)$ is a discrete version of the group of $2 \times 2$ matrices with real entries and determinant one-the special linear group, $\operatorname{Sl}(2, \mathbb{R})$, which has a similar action on the upper-half complex plane, (A.2). With this action $S l(2, \mathbb{R})$ maps semi-circles centred on the real line onto other semi-circles with the same property and obviously the discrete sub-group $\Gamma$ (1) must share this feature.

Clearly $\Gamma(1)$ maps rational numbers on the real line $\sigma=p / q$ to other rational numbers $\sigma=(a p+d q) /(c p+d q)$. A general element of $\Gamma(1)$ does not necessarily 
preserve the parity (even or odd) of the denominator under such action, $c p+d q$ can be either even or odd regardless of the parity of $q$, and so $\Gamma(1)$ itself can hardly be a symmetry group between QH states, $\sigma=\nu=p / q$, for which $q$ must be odd. However, the sub-group of $\Gamma(1)$ whose bottom left entry is even does preserve the parity of the denominator, and the possible significance of this property for the $\mathrm{QH}$ effect was first noticed by Lütken and Ross in [2]. This sub-group is often denoted by $\Gamma_{0}(2)$ in the mathematical literature (though Ref. [21] denotes it by $\Gamma_{U}(2)$ ) and it can be represented by matrices of the form

$$
\gamma=\left(\begin{array}{cc}
a & b \\
2 c & d
\end{array}\right) \quad \text { with } a d-2 b c=1,
$$

with $a, b, c$ and $d$ integers. $\Gamma_{0}(2)$ is generated by two elements $U: \sigma \rightarrow \sigma+1$ with

$$
g_{U}=\left(\begin{array}{ll}
1 & 1 \\
0 & 1
\end{array}\right)
$$

and $X: \sigma \rightarrow \sigma /(2 \sigma+1)$ with

$$
g_{X}=\left(\begin{array}{ll}
1 & 0 \\
2 & 1
\end{array}\right)
$$

These give a generalisation [6] to the whole upper-half complex plane of the Landau level addition transformation and the flux attachment rule respectively of Kivelson, Lee and Zhang [8], which were originally defined for rational filling factors only, corresponding to $\mathrm{QH}$ states.

This action of $\Gamma_{0}(2)$ on the upper-half $\sigma$-plane results in an inhomogeneous tiling of the plane - the whole plane can be generated by the action of $\Gamma_{0}(2)$ on a "fundamental domain", which can be taken to be a connected domain [21]. The definition of the fundamental domain is not unique, but a convenient choice is a vertical strip of unit radius extending infinitely far in the imaginary direction, but with its lower edge bounded by a semi-circular arch of unit diameter connecting the points $\sigma=0$ and $\sigma=1$ (see Fig. 2). The properties of any function are uniquely determined by knowledge of the function on the fundamental domain alone.

Since $\Gamma_{0}(2)$ is a sub-group of the full modular group it maps semi-circles centred on the real line onto other semi-circles with the same property. In particular the above mentioned semi-circular arch of radius $\frac{1}{2}$ spanning $\sigma=0$ and $\sigma=1$ is mapped by

$$
\gamma=\left(\begin{array}{cc}
a & b \\
2 c & d
\end{array}\right)
$$

into an arch of radius $1 /[2 d(2 c+d)]$, spanning $\sigma=b / d$ and $\sigma=(a+b) /(2 c+d)$. If $b / d=p_{1} / q_{1}$ and $(a+b) /(2 c+d)=p_{2} / q_{2}$, with $q_{1}$ and $q_{2}$ odd, are two QH states between which a transition is allowed we have $a=p_{1}-p_{2}, b=p_{1}, 2 c=q_{2}-q_{1}$ and $d=q_{1}$ so

$$
\gamma=\left(\begin{array}{ll}
p_{2}-p_{1} & p_{1} \\
q_{2}-q_{1} & q_{1}
\end{array}\right)
$$


Now the condition $\operatorname{det} \gamma=1 \Longleftrightarrow p_{2} q_{1}-p_{1} q_{2}=1$ which gives a selection rule for $\mathrm{QH}$ transitions [6]. In this way all allowed $\mathrm{QH}$ transitions can be generated from a "fundamental" transition describing crossover between the insulating state, with $\sigma=0$, and the QH state with filling factor unity, with $\sigma=1, \nu: 0 \rightarrow 1$, by the action of some element of $\Gamma_{0}(2)$. This immediately implies the hypothesis of universality of $\mathrm{QH}$ transitions [13].

The fixed points of $\Gamma_{0}(2)$ play an important rôle in crossover phenomena. The point $\sigma_{c}=\frac{1+i}{2}$ is left invariant by the element

$$
\gamma_{c}=\left(\begin{array}{ll}
1 & -1 \\
2 & -1
\end{array}\right)
$$

and there exist elements of $\Gamma_{0}(2)$ which leave any image, $\gamma\left(\sigma_{c}\right)$, of $\sigma_{c}$ fixed, namely $\gamma \gamma_{c} \gamma^{-1}\left\{\gamma\left(\sigma_{c}\right)\right\}=\gamma\left(\sigma_{c}\right)$. Under the assumption that the action of $\Gamma_{0}(2)$ on the $\sigma$ plane commutes with the RG flow, $\sigma_{c}$ and all its images must be fixed points of the RG flow $[2,5,6]$.

The Jacobi functions $\vartheta$-functions used in the text are defined by

$$
\begin{aligned}
& \vartheta_{2}=2 \sum_{n=0}^{\infty} q^{\left(n+\frac{1}{2}\right)^{2}}=2 q^{\frac{1}{4}} \prod_{n=1}^{\infty}\left(1-q^{2 n}\right)\left(1+q^{2 n}\right)^{2}, \\
& \vartheta_{3}=\sum_{n=-\infty}^{\infty} q^{n^{2}}=\prod_{n=1}^{\infty}\left(1-q^{2 n}\right)\left(1+q^{2 n-1}\right)^{2}, \\
& \vartheta_{4}=\sum_{n=-\infty}^{\infty}(-1)^{n} q^{n^{2}}=\prod_{n=1}^{\infty}\left(1-q^{2 n}\right)\left(1-q^{2 n-1}\right)^{2},
\end{aligned}
$$

where $q:=\mathrm{e}^{i \pi \sigma}$ (the conventions are those of [25], except that $\tau$ there is replaced by $\sigma$ here).

The $\vartheta$-functions satisfy the relation

$$
\vartheta_{3}^{4}=\vartheta_{2}^{2}+\vartheta_{4}^{4}
$$

and have the following transformations under $U: \sigma \rightarrow \sigma+1$ and $V: \sigma \rightarrow-1 / \sigma$ :

$$
\begin{array}{ll}
\vartheta_{2}(\sigma+1)=\mathrm{e}^{\frac{i \pi}{4}} \vartheta_{2}(\sigma), & \vartheta_{2}\left(-\frac{1}{\sigma}\right)=\sqrt{-i \sigma} \vartheta_{4}(\sigma), \\
\vartheta_{3}(\sigma+1)=\vartheta_{4}(\sigma), & \vartheta_{3}\left(-\frac{1}{\sigma}\right)=\sqrt{-i \sigma} \vartheta_{3}(\sigma), \\
\vartheta_{4}(\sigma+1)=\vartheta_{3}(\sigma), & \vartheta_{4}\left(-\frac{1}{\sigma}\right)=\sqrt{-i \sigma} \vartheta_{2}(\sigma)
\end{array}
$$

(a demonstration of these transformation properties can be found in [25]).

It is not difficult to verify, using the above relations, that the function

$$
f(\sigma)=-\frac{\vartheta_{3}^{4} \vartheta_{4}^{4}}{\vartheta_{2}^{8}}=-\frac{1}{256 q^{2}} \prod_{n=1}^{\infty} \frac{\left(1-q^{4 n-2}\right)^{8}}{\left(1+q^{2 n}\right)^{16}}
$$


is invariant under the above transformations $U$ and $X$, and hence invariant under all of $\Gamma_{0}(2)$.

The Jacobi $\vartheta$-functions satisfy the following differential equations (see Ref. [21], p. 231, Eq. (7.2.17)):

$$
\frac{\vartheta_{3}^{\prime}}{\vartheta_{3}}-\frac{\vartheta_{4}^{\prime}}{\vartheta_{4}}=\frac{i \pi}{4} \vartheta_{2}^{4}, \quad \frac{\vartheta_{2}^{\prime}}{\vartheta_{2}}-\frac{\vartheta_{3}^{\prime}}{\vartheta_{3}}=\frac{i \pi}{4} \vartheta_{4}^{4}, \quad \frac{\vartheta_{2}^{\prime}}{\vartheta_{2}}-\frac{\vartheta_{4}^{\prime}}{\vartheta_{4}}=\frac{i \pi}{4} \vartheta_{3}^{4},
$$

where ${ }^{\prime}=d / d \sigma$. Using these to differentiate (A.7) one finds

$$
\frac{d f}{d \sigma}=-i \pi\left(\vartheta_{3}^{4}+\vartheta_{4}^{4}\right) f
$$

In the text the following asymptotic forms of the $\vartheta$-functions are required, which are easily verified from the definitions (A.4):

$$
\text { (i) } \sigma \rightarrow i \infty: \quad \vartheta_{2} \approx 2 \mathrm{e}^{\frac{i \pi \sigma}{4}} \rightarrow 0, \quad \vartheta_{3} \rightarrow 1, \quad \vartheta_{4} \rightarrow 1, \quad f \approx-\frac{\mathrm{e}^{-2 \pi i \sigma}}{256} \rightarrow-\infty
$$

From these one can deduce, using (A.6),

$$
\text { (ii) } \begin{aligned}
\sigma \rightarrow 0: \quad \vartheta_{2} & \approx \sqrt{\frac{i}{\sigma}}, \quad \vartheta_{3} \approx \sqrt{\frac{i}{\sigma}}, \quad \vartheta_{4} \approx 2 \sqrt{\frac{i}{\sigma}} \mathrm{e}^{-\frac{i \pi}{4 \sigma}} \rightarrow 0, \\
f & \approx-16 \mathrm{e}^{-i \pi / \sigma} \rightarrow 0 .
\end{aligned}
$$

The behaviour of $f$ near $\sigma_{c}=\frac{1+i}{2}$ is also needed in the text. The following properties can be proven using the relation between $\vartheta$-functions and elliptic integrals given below (A.16),

$$
\vartheta_{3}^{4}\left(\sigma_{c}\right)=-\vartheta_{4}^{4}\left(\sigma_{c}\right)=\frac{i}{4 \pi^{3}}\left\{\Gamma\left(\frac{1}{4}\right\}^{4},\right.
$$

(where $\Gamma\left(\frac{1}{4}\right) \approx 3.626$ is the usual Gamma function) from which we can conclude, using (A.5), that

$$
\vartheta_{2}^{4}\left(\sigma_{c}\right)=2 \vartheta_{3}^{4}\left(\sigma_{c}\right) \Rightarrow f\left(\sigma_{c}\right)=\frac{1}{4} .
$$

With the help of (A.9) above, a Taylor expansion for $f(\sigma)$ around $\sigma_{c}=\frac{1+i}{2}$ can now be developed, using (A.8), (A.12) and (A.13),

$$
\begin{aligned}
f\left(\sigma_{c}+\varepsilon\right) & =\frac{1}{4}\left\{1-\left.2 i \pi \vartheta_{3}^{4}\left(\sigma_{c}\right)\left(\frac{\vartheta_{3}^{\prime}}{\vartheta_{3}}-\frac{\vartheta_{4}^{\prime}}{\vartheta_{4}}\right)\right|_{\sigma_{c}} \varepsilon^{2}+\ldots\right\} \\
& =\frac{1}{4}\left\{1+\frac{\pi^{2}}{2} \vartheta_{3}^{4}\left(\sigma_{c}\right) \vartheta_{2}^{4}\left(\sigma_{c}\right) \varepsilon^{2}+\ldots\right\} \\
& =\frac{1}{4}-\frac{1}{64 \pi^{4}}\left\{\Gamma\left(\frac{1}{4}\right)\right\}^{8} \varepsilon^{2}+\ldots
\end{aligned}
$$

The Jacobi $\vartheta$-functions are related to complete elliptic integrals of the second kind, 


$$
K(k)=\int_{0}^{\pi / 2} \frac{d \varphi}{\sqrt{1-k^{2} \sin ^{2} \varphi}}
$$

by the following formula (see Ref. [25] p. 479):

$$
\vartheta_{2}=\sqrt{\frac{2 k K(k)}{\pi}}, \quad \vartheta_{3}=\sqrt{\frac{2 K(k)}{\pi}}, \quad \vartheta_{4}=\sqrt{\frac{2 k^{\prime} K(k)}{\pi}},
$$

where the modulus $k$ is related to $\sigma$ by $\mathrm{e}^{-\pi K^{\prime} / K}=\mathrm{e}^{i \pi \sigma}$ and $K^{\prime}(k)=K\left(k^{\prime}\right)$, with $\left(k^{\prime}\right)^{2}:=1-k^{2}$ the complementary modulus.

The elliptic integral $K(k)$ has the following expansions (see Ref. [31], Eqs. (8.113.1) and (8.113.3)):

$$
\begin{aligned}
& K(k)=\frac{\pi}{2}\left(1+\frac{1}{4} k^{2}+\ldots\right), \quad|k| \ll 1, \\
& K(k)=\ln \left(\frac{4}{k^{\prime}}\right)+\frac{1}{4}\left\{\ln \left(\frac{4}{k^{\prime}}\right)-1\right\}\left(k^{\prime}\right)^{2}+\ldots, \quad\left|k^{\prime}\right| \ll 1 .
\end{aligned}
$$

Thus

$$
K^{\prime}(k)=\ln \left(\frac{4}{k}\right)+\frac{1}{4}\left\{\ln \left(\frac{4}{k}\right)-1\right\} k^{2}+\ldots, \quad|k| \ll 1 .
$$

In addition the following relations are needed in the text:

$$
\begin{aligned}
K\left(\frac{1}{k}\right) & =k\left\{K(k)+i K^{\prime}(k)\right\}, \\
K\left(i \frac{k}{k^{\prime}}\right) & =k^{\prime} K(k),
\end{aligned}
$$

(see Ref. [31], Eqs. (8.128.1) and (8.128.3): beware of the misprint in Eq. (8.128.3) of the fourth edition).

Finally, using (8.129.1) of [31],

$$
K\left(\frac{1}{\sqrt{2}}\right)=\frac{1}{4 \sqrt{\pi}}\left\{\Gamma\left(\frac{1}{4}\right)\right\}^{2}
$$

and (A.19) with (A.16) reproduces Eq. (A.12) above, since $\sigma=\frac{1+i}{2}$ corresponds to $k=\sqrt{2}$.

\section{References}

| I| C.A. Lütken and G.G. Ross, Phys. Rev. B 45 (1992) 11837.

[2] C.A. Lütken and G.G. Ross, Phys. Rev. B 48 ( 1993) 2500.

[3] A. Shapere and F. Wilczek, Nucl. Phys B 320 (1989) 669.

141 Y. Georgelin and J-C. Wallet, Phys. Lett. A 224 (1997) 303;

Y. Georgelin, T. Masson and J-C. Wallet, J. Phys. A 30 (1997) 5065. 
[5] C.A. Luitken, Nucl. Phys. B 396 (1993) 670.

16] B.P. Dolan, Duality and the modular group in the quantum Hall effect (cond-mat/9805171).

171 A.M. Dykhne and I.M. Ruzin, Phys. Rev. B 50 (1994) 2369;

I. Ruzin and S. Feng, Phys. Rev. Lett. 74 (1995) 154.

[8| S. Kivelson, D.-H. Lee and S-C. Zhang, Phys. Rev. B 46 (1992) 2223.

[9] C.P. Burgess and C.A. Lütken, Nucl. Phys. B 500 (1997) 367.

1101 A. Ritz, Phys. Lett. B 434 (1998) 54 (hep-th/9710112).

(11) J.I. Latorre and C.A. Lïtken, Phys. Lett. B 421 ( 1998) 217, (hep-th/9711150).

[12] D. Shahar, D.C. Tsui, M. Shayegan, E. Shimshoni and S.L. Sondhi, Phys. Rev. Lett. 79 (1997) 479, (cond-mat/9611011).

[13] A.M.M. Pruisken, Phys. Rev. Lett. 61 (1988) 1297.

$114 \mid$ D. Shahar, D.C. Tsui, M. Shayegan, J.E. Cunningham, E. Shimshoni and S.L. Sondhi, Solid State Comm. 102 (1997) 817 (cond-mat/9607127);

D. Shahar, D.C. Tsui, M. Shayegan, E. Shimshoni and S.L. Sondhi, Science 274 (1996) 589 (condmat/9510113).

|15] D.E. Khmel'nitskii, Pis'ma Zh. Eksp. Teor. Fiz 38 (1983) 454; JETP Lett. 38 ( 1983 ) 552.

| $16 \mid$ S.L. Sondhi, S.M. Girvin, J.P. Carini and D. Shahar, Rev. Mod. Phys. 69 (1997) 315.

|17| The Quantum Hall Effect, ed. R.E. Prange and S.M. Girvin (Springer, Berlin, 1987).

|18| N. Seiberg and E. Witten, Nucl. Phys. B 426 (1994) 19, (hep-th/9407087).

| $19 \mid$ C. Montonen and D. Olive, Phys. Lett. B 72 (1997) 117.

|20| J.L. Cardy and E. Rabinovici, Nucl. Phys. B 205 (1982) 1; J.L. Cardy, Nucl. Phys. B 205 (1982) 17.

121 ] R.A. Rankin, Modular Forms and Functions (Cambridge Univ. Press, Cambridge, 1977).

|22| P.H. Damgaard and P.E. Haagensen, J. Phys. A 30 (1997) 4681 (cond-mat/9609242).

[23] K. Yang, D. Shahar, R.N. Bhatt, D.C. Tsui and M. Shayegan, cond-mat/9805341.

[24] Y. Huo. R.E. Hetzel and R.N. Bhatt, Phys. Rev. Lett. 70 (1993) 481.

125| E.T. Whittaker and G.N. Watson, A Course of Modern Analysis (Cambridge Univ. Press, Cambridge, 1940).

|26] D. Shahar, M. Hilke, C.C. Li, D.C. Tsui, S.L. Sondhi and M. Razeghi, cond-mat/9706045.

[27] M. Hilke, D. Shahar, S.H. Song, D.C. Tsui, Y.H. Xie and D. Monroe, cond-mat/9708239.

|28] S.C. Zhang, Int. J. Mod. Phys. B 6 (1992) 25.

129| N. Taniguchi, Non-perturbative renormalization group functon for quantum Hall plateau transitions imposed by global symmetries, (cond-mat/9810334).

$130 \mid$ C.P. Burgess and C.A. Lütken, On the implications of discrete symmetries for the beta function of quantum Hall systems, (cond-mat/9812396).

131) I.S. Gradshteyn and I.M. Ryzhik, Tables of Integrals, Series and Products, 5th edition (Academic Press, New York, 1994). 